\title{
Incidencia de lesión renal en prematuros menores de 1,500 gramos en una Unidad Neonatal Colombiana
}

\author{
Pablo Vásquez-Hoyos, ${ }^{1, *}$ Tatiana González-Clavijo, ${ }^{1}$ Diana Paola Méndez-García, ${ }^{1}$ \\ Isabel Monje-Cardona, ${ }^{1}$ Nathalia Saavedra-Soler ${ }^{2}$ \\ ${ }^{1}$ Fundación Universitaria de Ciencias de la Salud, Hospital de San José; ${ }^{2}$ Hospital Infantil Universitario de San José, Bogotá, \\ Colombia.
}

\begin{abstract}
RESUMEN
Introducción: La lesión renal aguda (LRA) en recién nacidos $(\mathrm{RN})$ pretérmino es una complicación poco estudiada; en la actualidad no se cuenta con criterios para su definición y manejo. Objetivo: Determinar la incidencia de LRA en pacientes pretérmino hospitalizados en una unidad de cuidado neonatal (UCIN). Material y métodos: Estudio de cohorte descriptivo de $R N$ con peso $\leq 1,500$ gramos admitidos a UCIN de un hospital universitario en Bogotá, en un periodo de un año. A todos los pacientes se les tomó muestras de creatinina sérica a las 24 y 72 horas de vida. Se definió LRA como un incremento de creatinina $\geq 0.3 \mathrm{mg} / \mathrm{mL}$ o un aumento del $150 \%$ del valor inicial. Resultados: Se incluyeron 53 pacientes. Fueron excluidos 11 casos porque fallecieron antes de las 72 horas de nacidos. Diez casos cumplieron con la definición y corresponden a una incidencia de $23.8 \%$ (IC95\% 11.7 a $34.6 \%$ ). Hubo tres casos que también presentaron oliguria. Conclusiones: Con nuestra definición se evidencia una elevada incidencia de LRA en RN prematuros. Se requieren más estudios para confirmar estos hallazgos y establecer factores de riesgo asociados a su presentación.
\end{abstract}

Palabras clave: Lesión renal aguda, prematuro, creatinina, incidencia.

\begin{abstract}
Introduction: There are few studies on acute kidney injury (AKI) in preterm infants; currently, there are no criteria for its definition and management. Objective: To determine the incidence of AKI in preterm infants hospitalized in a neonatal care unit (NICU). Material and methods: Descriptive cohort study of newborns weighing $\leq 1,500$ grams admitted to the NICU of a university hospital in Bogotá, over a period of one year. All the patients were sampled serum creatinine at 24 and 72 hours of life. ARI was defined as an increase of creatinine $\geq 0.3 \mathrm{mg} / \mathrm{mL}$ or increased $150 \%$ of the initial value. Results: 53 patients were included. Eleven patients were excluded because they died before 72 hours after birth. Ten patients met the definition of ARI and correspond to an incidence of $23.8 \%(95 \% \mathrm{Cl} 11.7$ to $34.6 \%)$. There were three cases that also presented oliguria. Conclusions: Our definition shows a high incidence of AKI in premature newborns. More studies are needed to confirm these findings and establish risk factors associated with $\mathrm{AKI}$.
\end{abstract}

Keywords: Acute renal injury, premature, creatinine, incidence.

\footnotetext{
* Correspondencia: PVH, pvasquez@fucsalud.edu.co Conflicto de intereses: Los autores declaran que no tienen. Citar como: Vásquez-Hoyos P, González-Clavijo T, Méndez-García DP, Monje-Cardona I, Saavedra-Soler N. Incidencia de lesión renal en prematuros menores de 1,500 gramos en una Unidad Neonatal Colombiana. Rev Mex Pediatr. 2019; 86(5):181-184. [Incidence of acute renal injury in prematures under 1500 grams in a Neonatal Colombian Unit]
}

\section{INTRODUCCIÓN}

La lesión renal aguda (LRA) es una enfermedad producida por la disminución brusca del filtrado glomerular que ocasiona la retención de productos nitrogenados, aumento de la creatinina plasmática y disminución del volumen urinario, aunque también puede presentarse con volúmenes urinarios normales. ${ }^{1,2}$ Es frecuente su presentación en las unidades de cuidado neonatal, principalmente en los recién nacidos $(\mathrm{RN})$ pretérmino quienes por su alto con- 
tenido de agua corporal e inmadurez tubular renal muestran LRA no oligúrica, ${ }^{2-4}$ por lo cual puede pasar desapercibido en fases tempranas si no se realiza un seguimiento de la creatinina sérica.

En cuanto a su definición, actualmente no hay un consenso; la mayoría de los autores utiliza los incrementos de creatinina en muestras seriadas con respecto al valor inicial evaluado. ${ }^{5-7}$ Lo anterior se debe a la alta frecuencia de lesión renal no oligúrica ${ }^{2-4}$ y a que usualmente los valores de creatinina en los primeros días son los de la madre. . $^{1,8,9}$

Dada esta dificultad en la definición, es probable la amplia variación de la incidencia de LRA. ${ }^{1,7}$ En prematuros, son pocos los estudios y la incidencia oscila entre el 12 y $28 \%$.,6,10 Durante el desarrollo de esta investigación, se encontraron tres estudios, dos de ellos de recolección retrospectiva, con definiciones de incremento en creatinina y poblaciones menores de $1,500 \mathrm{~g}$ en los cuales se reportaron incidencias de 18 a $30 \% .{ }^{3,11,12} \mathrm{~A}$ la fecha de la realización de este estudio, no se encontró algún otro del mismo tipo en Latinoamérica.

El objetivo del estudio fue determinar la incidencia de LRA en prematuros que ingresan a una unidad de cuidado intensivo neonatal (UCIN) de un hospital universitario de la ciudad de Bogotá, Colombia.

\section{MATERIAL Y MÉTODOS}

Se realizó un estudio prospectivo y descriptivo en el que se incluyeron todos los $\mathrm{RN}$ pretérmino con muy bajo peso al nacer $(\leq 1,500 \mathrm{~g})$ que fueron hospitalizados en la UCIN del Hospital Infantil Universitario de San José, en el periodo comprendido entre marzo de 2016 y febrero de 2017 . El hospital es un centro de referencia para enfermedades materno-fetales y de alto riesgo de la ciudad de Bogotá.

Se excluyeron los prematuros que presentaban diagnóstico prenatal o sospecha clínica de aneuploidías, agenesia renal, masas renales o enfermedad poliquística renal. También se excluyeron pacientes que fallecieron en las primeras 72 horas de nacidos porque no fue posible tomar datos de creatinina seriada.

A todos los incluidos se les tomaron niveles de creatinina sérica a las 24 horas y 72 horas del nacimiento. Se recolectaron los datos de sexo, APGAR en el quinto minuto, peso y edad gestacional según la escala de Ballard. Además, se registró la presencia de preeclampsiaeclampsia, ruptura prematura de membranas, restricción del crecimiento intrauterino, estado fetal insatisfactorio y embarazo múltiple. También se documentaron factores de exposición para LRA en las primeras 72 horas de vida como ductus arterioso persistente con repercu- sión hemodinámica, uso de medicamentos vasoactivos y nefrotóxicos. Finalmente, se determinó la mortalidad y los días de estancia en la UCIN.

Se definió LRA como un incremento de creatinina sérica $>0.3 \mathrm{mg} / \mathrm{mL}$ respecto al valor basal o un aumento de $150 \%$ del valor inicial. Se midió la frecuencia de oliguria en las primeras 72 horas, que fue definida como gasto urinario de 24 horas $\leq 1 \mathrm{~mL} / \mathrm{kg} /$ hora, pero esto último no se utilizó como criterio diagnóstico de LRA.

El protocolo de investigación fue aprobado por el Comité de Ética del Hospital Infantil Universitario de San José.

Análisis estadístico. Fue de tipo descriptivo. Las variables cuantitativas se presentan con mediana y rangos intercuartílicos (RIQ) dado que no tuvieron distribución de normal. Para las cualitativas se usaron valores absolutos y porcentajes. Se calculó la incidencia acumulada de LRA a los siete días de vida con su

\section{Tabla 1: Características demográficas y factores de exposición en las primeras 72 horas de vida de 42 recién nacidos $\leq 1,500 \mathrm{~g}$.}

\begin{tabular}{l} 
Características \\
\hline Edad gestacional por Ballard \\
$\leq 28$ semanas \\
de 29 a 32 semanas \\
Peso \\
$\leq 1,000 \mathrm{~g}$ \\
$1,001-1,500 \mathrm{~g}$ \\
Sexo \\
Masculino \\
Femenino \\
Factor desencadenante de parto \\
Preeclampsia/eclampsia* \\
Ruptura prematura de membranas \\
Restricción de crecimiento intrauterino \\
Estado fetal insatisfactorio* \\
Embarazo múltiple \\
Exposición a nefrotóxicos \\
Paracetamol \\
Ibuprofeno \\
Amikacina \\
Otros factores \\
Vasoactivos \\
DAP con repercusión \\
Asfixia (Apgar $5^{\circ}$ minuto $\leq 5$ )
\end{tabular}

$n$

18

24

\section{7}

25

20

22

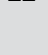

25

7

4

10

10

20

2

35

26

13

3

* Pacientes con dos factores desencadenantes. 
Tabla 2: Características individuales de los 10 prematuros con lesión renal aguda.

\begin{tabular}{|c|c|c|c|c|c|c|c|c|c|}
\hline ID & $\begin{array}{l}\text { Creatinina } \\
\text { inicial } \\
(\mathrm{mg} / \mathrm{dL})\end{array}$ & $\begin{array}{l}\text { Creatinina } \\
\text { a } 72 \text { horas } \\
(\mathrm{mg} / \mathrm{dL})\end{array}$ & Sexo & $\begin{array}{c}\text { Edad } \\
\text { gestacional } \\
\text { (semanas) }\end{array}$ & $\begin{array}{l}\text { Causa de parto } \\
\text { pretérmino }\end{array}$ & Asfixia & $\begin{array}{l}\text { Aminoglu- } \\
\text { cósido }\end{array}$ & Oliguria & $\begin{array}{l}\text { Supervivencia } \\
\text { (días) }\end{array}$ \\
\hline 1 & 0.62 & 1.13 & Masculino & 27 & Corioamnionitis & Sí & No & No & 4 \\
\hline 2 & 0.83 & 1.32 & Masculino & 29 & $\begin{array}{l}\text { Estado fetal insatis- } \\
\text { factorio, restricción de } \\
\text { crecimiento intrauterino }\end{array}$ & No & Amikacina & Sí & 4 \\
\hline 3 & 0.64 & 0.94 & Femenino & 29 & Preeclampsia & No & Amikacina & No & $37^{*}$ \\
\hline 4 & 0.52 & 0.85 & Masculino & 29 & Corioamnionitis & No & Amikacina & No & 6 \\
\hline 5 & 0.48 & 1.17 & Masculino & 30 & Eclampsia & No & Amikacina & No & 3 \\
\hline 6 & 0.67 & 2.27 & Femenino & 28 & $\begin{array}{l}\text { Ruptura prematura } \\
\text { de membranas }\end{array}$ & No & Amikacina & Sí & 3 \\
\hline 7 & 0.75 & 1.58 & Femenino & 28 & Preeclampsia & No & Amikacina & No & 3 \\
\hline 8 & 0.62 & 1.07 & Femenino & 26 & Embarazo múltiple & No & Amikacina & No & 13 \\
\hline 9 & 0.69 & 1.02 & Femenino & 25 & Embarazo múltiple & Sí & Amikacina & No & 7 \\
\hline 10 & 0.73 & 1.04 & Masculino & 24 & $\begin{array}{c}\text { Ruptura prematura de } \\
\text { membranas }\end{array}$ & No & Amikacina & Sí & 3 \\
\hline
\end{tabular}

respectivo intervalo de confianza del 95\% (IC95\%). Los datos fueron tabulados en Excel y analizados en el paquete estadístico Stata 13.

\section{RESULTADOS}

Durante el periodo de estudio, de un total de 4,338 nacimientos, 472 fueron hospitalizados en la UCIN, de los cuales, 53 prematuros cumplieron los criterios de inclusión. De éstos, once fueron excluidos porque fallecieron antes de la toma de creatinina sérica de control. No hubo pacientes con malformaciones o defectos congénitos.

Las características demográficas y factores de exposición de los 42 prematuros incluidos se muestran en la Tabla 1. Para resaltar, el $42.9 \%$ correspondió a prematuros extremos ( $\leq 28$ semanas) y $40.5 \%$ a peso extremadamente bajo al nacer $(\leq 1,000$ gramos). Al revisar el puntaje de APGAR a los cinco minutos se observó que tres pacientes presentaron un valor $\leq 5$ sugestivo de asfixia perinatal.

En cuanto a la exposición a agentes nefrotóxicos, el $4.8 \%$ recibió ibuprofeno y el $83.6 \%$ estuvo expuesto a amikacina. El 31\% tuvo ductus arterioso con repercusión hemodinámica y $61.9 \%$ requirió algún apoyo vasoactivo.

La mediana de estancia en la UCIN fue de 25 días (rango intercuartílico 5-36). La mortalidad general del grupo fue del $42.9 \%$ vida postnatal.
Se diagnosticó LRA en 10 casos (23.8\%), de los cuales siete $(70 \%)$ cumplieron con los dos criterios diagnósticos y tres cumplieron sólo con el aumento de $0.3 \mathrm{mg} / \mathrm{dL}$ de la creatinina basal. Durante las primeras 72 horas tres pacientes presentaron oliguria.

Las principales características demográficas de los 10 pacientes con LRA se describen en la Tabla 2. Se destaca que siete pacientes fueron prematuros extremos, ocho tuvieron un peso $\leq 1,000$ gramos, dos presentaron asfixia neonatal, dos con ductus arterioso y todos requirieron apoyo vasoactivo. $\mathrm{Al}$ final, nueve pacientes con LRA fallecieron.

\section{DISCUSIÓN}

Hasta nuestro conocimiento este es el primer estudio en Latinoamérica que reporta la incidencia de LRA en $\mathrm{RN} \leq 1,500 \mathrm{~g}$ en las primeras 72 horas de vida. Los resultados del presente estudio evidencian una incidencia similar a los estudios descritos en la literatura que utilizan la misma definición. ${ }^{6,11}$

El estudio de Koralkar de 2011 en Birmingham, Alabama $^{12}$ describió una cohorte prospectiva de $\mathrm{RN}$ $<1,500$ g con seguimiento hasta el egreso o 36 semanas postconcepcionales y reportó una incidencia del $18 \%$. Esta incidencia es un poco inferior a la de este estudio, lo cual puede deberse a que no utiliza un 
muestreo sistemático de creatinina, sino recolección por conveniencia de remanentes de suero o estudios solicitados por el grupo tratante, lo que pudo generar un subregistro. Askenazi ${ }^{11}$ en 2015 publicó en el mismo centro de Alabama un estudio en el que se realizó un seguimiento de $\mathrm{RN} \leq 1,200$ g y una recolección sistemática en los primeros cuatro días de vida, encontrando una incidencia mayor (30\%). Esta incidencia es un poco mayor a la del presente estudio, probablemente por ser una población más prematura e incluyeron pacientes con creatininas anormales hasta de 12 días de nacidos.

En Novi Sad, Serbia, el grupo de Stojanović en 2014 publicaron una serie retrospectiva de $\mathrm{RN} \leq 1,500 \mathrm{~g}$ con mediciones repetidas a partir del tercer día de vida, con un seguimiento hasta el séptimo día. La incidencia fue similar al presente estudio (26\%); sin embargo, las condiciones de vigilancia fueron diferentes. ${ }^{3}$

Los criterios de LRA, como la de pRIFLE o AKIN en pacientes que no son RN, incluye la oliguria. ${ }^{13} \mathrm{En}$ este estudio todos los pacientes con oliguria cumplieron criterios de cambio de creatinina sérica y representan el $30 \%$ de los casos. En el estudio de Stojanović, el 13\% de los casos con LRA presentaron oliguria. ${ }^{3}$ Los estudios de Alabama no reportaron la frecuencia de oliguria. ${ }^{11,12}$

Dentro de los factores asociados a LRA, en nuestro estudio hubo dos casos de asfixia perinatal. Esta asociación ha sido poco estudiada. Durkan ${ }^{14}$ realizó una revisión en 2011 en la que comenta la escasa cantidad de estudios disponibles y la heterogeneidad de las definiciones que limitan estimar la incidencia en este grupo.

También se ha estudiado el efecto de medicamentos nefrotóxicos como los aminoglucósidos en el desarrollo de la LRA. Para amikacina, específicamente en RN pretérmino, Giapros ${ }^{15}$ en 2001 comparó los niveles de creatinina antes y después de un tratamiento de siete días sin observar diferencias significativas; asimismo, tampoco hubo diferencias cuando se comparó entre quienes no recibieron amikacina. En este trabajo no se logró establecer la asociación, dado que la mayoría de los $\mathrm{RN}$ recibieron amikacina.

En cuanto a la supervivencia, los RN con LRA de esta cohorte tuvieron una alta mortalidad, ya que fue del $90 \%$, en comparación a estudios previos que la reportan con una variación de 22 a $69 \% .^{3,11,12}$ La mayor mortalidad en nuestra serie puede deberse a que fueron pacientes con LRA en las primeras 72 horas de vida, por lo que pueden ser pacientes más graves desde el nacimiento.

Si bien el presente estudio representa la primera cohorte latinoamericana que reporta la incidencia de LRA en prematuros y que evalúa prospectivamente esta complicación en las primeras 72 horas de vida, se debe reconocer sus limitaciones: la principal es el tamaño de la muestra que impidió la identificación de factores de riesgo de LRA, además que sólo fue de una UCIN, lo que acota su validez externa.

\section{AGRADECIMIENTOS}

Agradecemos al personal de enfermería, residentes, pediatras y neonatólogos de la UCIN del Hospital Infantil Universitario de San José por su colaboración en la recolección de las muestras. También agradecemos a Merideidy Plazas por su asesoría metodológica para el desarrollo de este estudio.

\section{REFERENCIAS}

1. Chua AN, Sarwal MM. Acute renal failure management in the neonate. NeoReviews. 2005; 6(8): e369-e376.

2. Mehta RL, Kellum JA, Shah SV, Molitoris BA, Ronco C, Warnock DG et al. Acute Kidney Injury Network: report of an initiative to improve outcomes in acute kidney injury. Crit Care. 2007; 11(2): R31.

3. Stojanović V, Barišić N, Milanović B, Doronjski A. Acute kidney injury in preterm infants admitted to a neonatal intensive care unit. Pediatr Nephrol. 2014; 29(11): 2213-2220.

4. Askenazi D, Saeidi B, Koralkar R, Ambalavanan N, Griffin RL. Acute changes in fluid status affect the incidence, associative clinical outcomes, and urine biomarker performance in premature infants with acute kidney injury. Pediatr Nephrol. 2016; 31(5): 843-851.

5. Chan JC, Williams DM, Roth KS. Kidney failure in infants and children. Pediatr Rev. 2002; 23(2): 47-60.

6. Daga A, Dapaah-Siakwan F, Rajbhandari S, Arevalo C, Salvador A. Diagnosis and risk factors of acute kidney injury in very low birth weight infants. Pediatr Neonatol. 2016.

7. Drukker A, Guignard JP. Renal aspects of the term and preterm infant: a selective update. Curr Opin Pediatr. 2002; 14(2): 175-182.

8. Kelly LK, Seri I. Renal developmental physiology: relevance to clinical care. NeoReviews. 2008; 9(4): e150-e161.

9. Ringer SA. Acute renal failure in the neonate. NeoReviews. 2010; 11(5): e243-e251.

10. Nagaraj N, Berwal PK, Srinivas A, Berwal A. A study of acute kidney injury in hospitalized preterm neonates in NICU. J Neonatal Perinatal Med. 2016; 9(4): 417-421.

11. Askenazi D, Patil NR, Ambalavanan N, Balena-Borneman J, Lozano DJ, Ramani $\mathrm{M}$ et al. Acute kidney injury is associated with bronchopulmonary dysplasia/mortality in premature infants. Pediatr Nephrol. 2015; 30(9): 1511-1518.

12. Koralkar R, Ambalavanan N, Levitan EB, McGwin G, Goldstein S, Askenazi $D$. Acute kidney injury reduces survival in very low birth weight infants. Pediatr Res. 2011; 69(4): 354-358.

13. Kavaz A, Ozçakar ZB, Kendirli T, Oztürk BB, Ekim M, Yalçinkaya F. Acute kidney injury in a paediatric intensive care unit: comparison of the pRIFLE and AKIN criteria. Acta Paediatr. 2012; 101(3): e126-129.

14. Durkan AM, Alexander RT. Acute kidney injury post neonatal asphyxia. J Pediatr. 2011; 158(2 Suppl): e29-33.

15. Giapros VI, Andronikou SK, Cholevas VI, Papadopoulou ZL. Renal function and effect of aminoglycoside therapy during the first ten days of life. Pediatr Nephrol. 2003; 18(1): 46-52. 